\title{
Control Algorithms and Control Strategies for Variable Primary Flow Chilled Water Systems
}

\author{
Jinming Yang \\ College of Environmental Science \& Engineering \\ Taiyuan University of Technology \\ Taiyuan, China \\ Jinming123@yahoo.com.cn
}

\author{
Yi Lin \\ Shanxi Lineng Technology co.,ltd \\ Taiyuan, China \\ Linyi1667@163.com
}

\begin{abstract}
In recent years, variable primary flow control technology for chilled water systems has played an important role in energy saving. How to design the control system with appropriate control algorithms control strategies is particularly important? This article described the prerequisite for the realization of variable flow technology and discusses the three aspects emphatically. We can clarify the basic control principle of variable primary flow technology based on analysis on DOF (degree of freedom) of chilled water systems, it is important for control system design. The article illustrated the synchronous frequency conversion technology for multiple chilled water pumps system to avoid overload when part of pumps put in operation, finally discussed control algorithms control strategies for variable primary flow system. The article proposed that the fuzzy control algorithm is the good choice, then explained the control strategies for judging to addsubtract chiller or pump based on the maximum and minimum operating frequency of chilled water pumps with a actual project.
\end{abstract}

Keywords- Variable primary flow, Chilled water systems, Degree of freedom analysis, Synchronous frequency conversion technology, Fuzzy control, Control algorithms and control strategies

\section{INTRODUCTION}

In the past few decades, due to limited manufacturing technology of chillers and control technology, for the protection of the safe running of the chillers, most of the conventional design of chilled water system use constant flow system or primary-secondary pumping system to ensure that the chilled water flow rate through the chiller is constant. The energy conservation benefit is poor even if the system uses primary-secondary pumping system. Now variable primary flow chilled water systems pumping system were used to reduce building energy consumption and meet the global energy shortage. In order to ensure the normal operation of the system, the corresponding control technology of variable flow chilled water system is particularly important, especially for multi-chillers systems. A good design not only brings energy conservation benefit for chilled water pumps, optimizes chillers operating conditions and thus saves chillers energy consumption, but also ensures the safe operation of the chillers, and reduces risks associated with the low flow rate through the chillers. We know that the flowrate of chilled water circulating pumps and capacity of chillers are designed according to design load, when the system is part load, the operating frequency of pumps, the numbers and the operating conditions of operating chillers will change. Energy conservation benefit is guaranteed together by the chillers and the chilled water pumps.

Realization of variable chilled water technology must have certain conditions, otherwise, it will cause the system not normal, or the indoor temperature and humidity do not meet the design parameters, or even lead to damage to the chiller. Comprehensive consideration of all factors, realizing that technology must meet the following conditions:

(1)Chillers have the good ability to adapt to variable primary flow chilled water system. Today, the most of chillers produced by the vast majority of manufacturers support the flow rate range from $40 \%$ to $110 \%$ through the evaporators, such as screw chiller, centrifugal chiller, compressor chillers and absorption liquid chillers. This is the prerequisite for the design of variable primary flow chilled water system.

(2) The chiller has the good load control ability. They should have the faster rate of change to respond to variable flow rate through the evaporator. Variable primary flow optimization of chiller offers the key to variable flow control.

(3) To obtain accurate system information, The system needs to use the temperature sensors and flow sensors with high-precision.

(4) We must analyze Degrees of Freedom (DoF) for a variable primary chilled water system before designing a control system, and then determine the controlled variables and manipulated variables, which is premise.

(5)Chilled water circulation pumps as far as possible use the synchronized frequency conversion technology for the multi pump system. All pumps must configure the frequency converters.

(6) The variable primary chilled water system uses the appropriate control algorithms and control strategies.

This article will discuss the final three viewpoints emphatically.

\section{DEGREES Of FreEdOM ANALYSIS Of CONTROL SYSTEM}

Theory about degrees of freedom is actually on the constraints of a physical system. DoF is defined as the number of independent variables relative to a certain process, 
in order to determine the process completely, which is expressed by the following formula:

$$
N_{f}=N_{V}-N_{e}
$$

In the above formula, $N_{f}$ is DoF, $N_{V}$ is number of independent variables for a process, and $N_{e}$ is number of independent equations related with independent variables. For the design of an effective control system, to ascertain the value of DoF for a certain process and which variables are DoF is important, only when DoF is determined completely can we achieve the desired process control. According to the theory of analysis for DoF, the process with numerous solutions is lack of stability when $N_{f}>0$, the process with no solutions is extremely stable when $N_{f}<0$, and process with unique solution is stable when $N_{f}=0$. For a process which DoF is greater than 0 , we must try to reduce its DoF to zero in order to assure the unique solution for the process.

We can use three ways to reduce the DoF, binding a variable to a constant by outer condition, listing some coupling equations between variables according to the theory about conservation of energy or quantities of objects, constructing a control loop by deciding a controlled variable. so when a variable is determined for the constant or when a equation is established or a control loop is constructed, DoF can be reduced one.

For chilled water system, based on the energy conservation law, we can list the only one equation below.

$$
Q=m c\left(t_{r}-t_{s}\right)
$$

In the formulas above:

$Q$ - the total cooling capcity output by the chillers;

$m-$ mass flow of chilled water system through the evaporators ;

$$
\begin{aligned}
& t_{r} \text {-return chilled water temperature; } \\
& t_{s-\text { supply chilled water temperature; }} \\
& c \text { - specific heat of water; }
\end{aligned}
$$

For the chilled water system, there are 5 independent process variables, with one of them $c$ is known variable and the others are unknown. So according to theory of DoF discussed above, the system has four independent process variables, and $N_{V}=4$.

$$
\text { We can list one equation (2) so } N_{e}=1 \text { and }
$$
$N_{f}=N_{V}-N_{e}=3$, The discussion above is just the premises for design of control system. In order to keep the stability of chilled water system, we must mange to reduce the value of DoF from 3 to zero. Usually we reduce DoF by assigning a fixed value to some variable or construct some control loops.

For the chilled water system, we can calculate the cooling load $Q$ for air conditioning with the data sampled last time. In other words cooling load is already determined and known at any moment, so $N_{f}=3-1=2$. To reduce the rest two DOF, we can construct two control loops, one is supply chilled water temperature control loop with supply water temperature is controlled variable which achieved by the control system of chillers, the other is return chilled water temperature control loop with return water temperature is controlled variable which achieved by the extra controllers such as PLC and Single-chip microcomputer, etc. The first loop ensure the supply temperature by changing the output of chillers, the second ensure return temperature by changing the flow rate .In fact when the $t_{r}$ and $t_{s}$ meet design values such as $t_{r}=12{ }^{\circ} \mathrm{C}$ and $t_{s}=7{ }^{\circ} \mathrm{C}$, we can think simply the indoor temperature meet requirement. so $N_{f}=2-1-1=0$ and the system has a unique solution[1].

\section{SyNCHRONIZED FREQUENCY CONVERSION TECHNOLOGY For Multi PumPS System}

Regarding actual project, the chilled water system is usually multiple parallel pumps system. How do we operate pumps when the system changes the chilled water flow? What's the best way to meet the system requirements? The answer is synchronous frequency conversion technology. All pumps should be run simultaneously with the same frequency. That is, we regard multiple pumps as a pump. In this way we can get maximum energy efficiency and safe operation of the chilled water system, Otherwise running pumps are at risk of overload when only part of pumps designed run. For example, When the water flow is half of the design flowrate for systems configured two operating pumps, two water pumps should put the operation simultaneously with operating frequency of $25 \mathrm{HZ}$, pumps flow and pump head match the pipeline characteristic, and the total electric power consumption is merely $1 / 4$ of the rated power of one pump. By contrast, the electric power consumption is close to the rated power when one pump only put the operation, it brings not only the more electric power consumption but also the risk of overload resulted from more flowrate exceed the demand. Principle discussed above is easy understand when we know the relationship between the characteristic of parallel pumps and pipeline characteristic.

\section{CONTROl Algorithms And CONTROL StRATEgIES}

In addition to some of the issues discussed above, there are other issues that must be concerned about, especially control algorithms and control strategies for variable primary flow chilled water systems. We know that PID control algorithm is the most classic algorithm in the actual control system, More than 95 per cent of actual projects use PID algorithm. But PID algorithm is not suitable for the chilled water system with the large capacity and long time-delay. We must introduce improved control algorithms such as feedforward control algorithm, cascade control algorithm and fuzzy control algorithm, etc. In which popular is the fuzzy control algorithm gradually. We know that supply chilled water control loop, one of control loops constructed 
based DOF theory, is achieved by chiller control system provided by the manufacturer. The other return chilled water control loop is the only loop we need to concern. How to ensure that return chilled water temperature meet the design value through the reasonable fuzzy control algorithm? The key is the construction of fuzzy control rules and Fuzzy Decision-making based on the expert knowledge. The fuzzy control system usually adopts double-variables model which is called Mamdani model as shown in the figure 1 below. In figure $1 e$ is the deviation of return water temperature and $e c$ is the return water temperature deviation rate of change, they are input variables. The chilled water flowrate $q$ is defined as the output variable. By using controllers with fuzzy control algorithm, we can ensure the value of returned chilled water temperature. Here we do not discuss fuzzy control theory in detail[2].

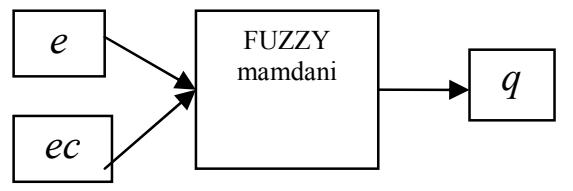

Figure 1. Mamdani model of fuzzy control

For multi-chillers and multi-pumps chilled water system we must be careful to take into account the design of control strategies, otherwise it will lead to control system failure or even result in a low supply water temperature risk of chillers. The control system should decide the way to add-subtract chiller and add-subtract pump according to the air conditioning load for the building.

It is necessary to add-subtract chiller with change of air conditioning load for multiple chillers system. First of all we must construct the rules to add-subtract chiller. Some chillers provide signal interface corresponding operating conditions such as RLA\%(Rated Load Amps), the ratio of operating load amps and rated load amps. With the signal, control system can judge the operating conditions of chillers, then decide when and how to add-subtract chiller. But some chillers have not such signal interface, we should develop a new control strategy for judging how to add-subtract chiller, which is called judgmental method to add-subtract chiller based on the maximum and minimum operating frequency of chilled water circulating pumps. As the air conditioning load increases, the supply chilled water temperature and return water temperature begin to rise, so the control loops put into operation immediately. The operating pumps chillers will increase their speed in order to supply more flowrate and ensure the pure value of return temperature to meet setpoint value, consequently chillers detect a sensible rise in supply water temperature caused by the increased flowrate, and increase their output to ensure the pure value of supply temperature to meet setpoint value. The two control loops work separately, when the operating pumps reach the maximum operating frequency, the control system can judge operating conditions of chillers by sampling supply water temperature, if pure value is higher than setpoint value and this situation continued for certain time such as several 10 minutes, the control system can judge that it's time to put a chiller into operation. On the contrary, when the operating pumps reach the minimum operating frequency(the flowrate corresponding to the minimum frequency must be greater than the permissible value of chiller), the control system can judge operating conditions of chillers by sampling supply water temperature also, if pure value is higher than setpoint value and this situation continued for certain time, the control system can judge that it's time to stop a operating chiller. They are control strategies for judging to addsubtract chiller based on the maximum and minimum operating frequency of chilled water pumps. This method does not require additional conditions for the chiller.

As is mentioned above, all chilled water pumps should be put into operation simultaneously with the same frequency for the multiple pumps system, the synchronous frequency conversion technology is is the preferred choice for the system with a smaller number of parallel pumps. But sometimes for the water system with a large number of parallel pumps, this approach has limitations. Lower operating frequency may bring risk to pumps and frequency converter, to avoid the adverse impact of low operating frequency, control system should limit the minimum operating frequency, such as $25 \mathrm{HZ}$. The synchronous frequency conversion technology causes a new problem, the article illustrates it with an actual project which configured four chillers and four chilled water pumps. It is no problem when the air conditioning load is greater than half of design load, all four pumps is running with same frequency(higher than the minimum frequency $25 \mathrm{HZ}$ ). When the load drops to less than half of the design load, such as quarter of design value, and only one chiller is operating, pump frequency should be less than $25 \mathrm{HZ}$ (theoretical value is $12.5 \mathrm{HZ}$ ), this is obviously not reasonable. The method to add-subtract pump should be adopted, and operating principle is similar to the method to add-subtract chiller. When the operating pumps reach the minimum operating frequency, such as $25 \mathrm{HZ}$, the control system can judge system conditions by sampling return water temperature also, if pure value is lower than setpoint value and this situation continued for certain time, the control system can judge that it's time to stop a operating pump, and vice versa. When only portion of pumps put in operation, what calls for special attention is that the maximum frequency of pumps should be lower than allowable frequency to avoid overload, such as $45 \mathrm{HZ}$ or lower. The fewer the number of pumps put in operation, the lower upper limit frequency[3].

\section{SUMMARIES}

We should know the prerequisite for the realization of variable flow technology before designing control system. It is important for control system design that we clarify the basic control principle of variable primary flow technology based on analysis on DOF of chilled water systems. The synchronous frequency conversion technology for multiple chilled water pumps system is a good choice to avoid overload. The article proposed that the fuzzy control algorithm is the good choice, further more the control system 
can adopt a new method to add-subtract chiller or pump based on the maximum and minimum operating frequency of chilled water pumps even for the chiller with no necessary signal interface, it is helpful for project practice.

\section{REFERENCES}

[1] Huihe Shao. Advanced Control of Industrial Process. CHN: Shanghai Jiao Tong University Press, 2003, pp. 5-10.
[2] Jinming Yang. Variable Flow Technique for Heating Circulating Pump.ICCASM 2010[2010 International Conference on Computer application and System Modeling] V11-307

[3] Jinming Yang. Management and control for Optimal Performance of the Heating Substation, ICEBO2006, Shenzhen,China, vol VI-7-5, November 2006[International Conference for Enhanced building Operations Committee,2006] 